\title{
ARTICLE
}

Received 13 Mar 2013 | Accepted 2 Aug 2013 | Published 6 Sep $2013 \quad$ DOl: 10.1038/ncomms3399

\section{Identification of a splice variant of mouse TRPA1 that regulates TRPA1 activity}

Yiming Zhou', Yoshiro Suzuki ${ }^{1,2}$, Kunitoshi Uchida ${ }^{1} \&$ Makoto Tominaga ${ }^{1,2}$

Transient receptor potential ankyrin 1 (TRPA1) protein is a nonselective cation channel. Although many studies suggest that TRPA 1 is involved in inflammatory and neuropathic pain, its mechanism remains unclear. Here we identify an alternative splice variant of the mouse Trpa1 gene. TRPA1a (full-length) and TRPA1b (splice variant) physically interact with each other and TRPA1b increases the expression of TRPA1a in the plasma membrane. TRPA1a and TRPA1b co-expression significantly increases current density in response to different agonists without affecting their single-channel conductance. Exogenous overexpression of Trpalb gene in wild-type and TRPA1KO DRG neurons also increases TRPA1a-mediated AITC responses. Moreover, expression levels of Trpala and Trpalb mRNAs change dynamically in two pain models (complete Freund's adjuvant-induced inflammatory pain and partial sciatic nerve ligation-induced neuropathic pain models). These results suggest that TRPA1 may be regulated through alternative splicing under these pathological conditions.

\footnotetext{
${ }^{1}$ Division of Cell Signaling, Okazaki Institute for Integrative Bioscience (National Institute for Physiological Sciences), National Institutes of Natural Sciences, Okazaki, Japan. ${ }^{2}$ Department of Physiological Sciences, The Graduate University for Advanced Studies, Okazaki, Japan. Correspondence and requests for materials should be addressed to M.T. (email: tominaga@nips.ac.jp).
} 
T ransient receptor potential (TRP) ion channels are nonselective cation channels that were originally discovered in mutant Drosophila ${ }^{1}$. Many studies revealed that the TRP channel superfamily is important for sensations such as taste, pain and temperature, both in the peripheral and central nervous systems ${ }^{2,3}$. The TRP channel superfamily is now divided into seven subfamilies: TRPV (Vanilloid), TRPC (Canonical), TRPM (Melastatin), TRPML (Mucolipin), TRPN (NompC), TRPP (Polycystic) and TRPA (Ankyrin). TRPA1 is the only member of the TRPA subfamily in mammals, with the presence of up to 17 ankyrin repeat domains ${ }^{4}$. TRPA1 channel is predominantly expressed in sensory neurons ${ }^{3}$, while there is some expression in other cell types such as astrocytes, enterochromaffin cells and pancreatic $\beta$-cells ${ }^{5-7}$. In sensory neurons of the dorsal root ganglion (DRG) and trigeminal ganglion (TG), TRPA1 channels are predominately expressed in small-diameter neurons. They are believed to be involved in many forms of nociception, with some reports suggesting that nerve growth factor regulates chronic inflammatory hyperalgesia by controlling Trpa1 gene expression ${ }^{8}$.

Alternative splicing of pre-mRNA was identified more than 30 years ago when it was discovered that two proteins could be encoded by the same gene $e^{9,10}$. Alternative splicing is a complex regulatory mechanism in eukaryotes by which protein diversity is increased from a relatively small number of genes ${ }^{11}$. More than 95\% of human genes are estimated to undergo alternative splicing ${ }^{12,13}$. Alternative splicing also has an important role during cell differentiation and development in a time- and tissuespecific manner ${ }^{14,15}$. Recently, two studies identified several alternative splice variants of $\operatorname{TrpA1}$ in Drosophila that were expressed in a tissue-specific manner and displayed different chemical and thermal sensitivities ${ }^{16,17}$. However, to date, there has been no reports of a mammalian TRPA1 splice variant, even though there are some instances of other TRP channels undergoing alternative splicing ${ }^{18,19}$. For instance, an alternative splice variant of $\operatorname{Trpv1}$ was reported to be a dominant-negative isoform ${ }^{20}$.

In this study, a splice variant of the mouse Trpal gene is identified and cloned from DRG neurons. This splice variant skips exon 20, which encodes 30 amino acids. The full-length TRPA1 protein is designated TRPA1a and the splice variant as TRPA1b. TRPAla and TRPA1b physically interact with each other. Co-expression of TRPAla with TRPAlb significantly increases TRPA1 agonist responses. Moreover, dynamic changes in Trpala and Trpalb expression levels are observed under inflammatory and neuropathic pain conditions. These results suggest TRPA1 might be involved in these pathologic conditions through alternative splicing.

\section{Results}

Cloning a mouse Trpal alternative splice variant. TRPA1 is predominantly expressed in DRG and TG neurons and functions as a nociceptive receptor ${ }^{3,21}$. Starting with RNA from mouse DRG neurons, RT-PCR analysis revealed an additional fragment using primers designed to amplify a partial fragment of mouse Trpa1 mRNA (Fig. 1a), suggesting that this additional fragment was an alternative splice variant. To confirm that this result was not due to non-specific amplification, two fragments were extracted from the agarose gel and sequenced. The additional fragment indeed corresponded to a Trpal splice variant whose sequence was confirmed by rapid amplification of cDNA ends using RNA from mouse DRG neurons. The splice variant excluded exon 20, which contained $90 \mathrm{bp}$. Full-length mRNA was referred to as Trpala and the variant as Trpalb (Fig. 1b). The amino-acid sequence of the TRPAla protein is identical to the previously reported TRPA1 channel that contains 1,125 amino acids $^{3}$. As there was no reading frame shifting, TRPAlb excluded only the sequence corresponding to the 30 amino acids from 777 to 807 in TRPAla, resulting in a protein of 1,095 amino acids. The deleted amino acids were in the second transmembrane domain and the first intracellular loop (Fig. 1c). Trpala and Trpalb mRNAs were expressed in DRG and TG as well as in small intestine and colon, although both Trpala and Trpalb expression in small intestine and colon was relatively weaker than that in DRG and TG (Fig. 1d). Single-cell RT-PCR analysis confirmed that Trpala and Trpalb were expressed in the same DRG neurons together with Trpv1 (Fig. 1e). Although the nucleotide sequence of exon 20 is highly conserved among mouse, and rat Trpal and human TRPA1 (Supplementary Fig. S1a), no additional RT-PCR fragment was amplified using rat and human RNAs, indicating that this splice variant could be mouse-specific (Supplementary Fig. S1b). Using DRG samples collected from 10 mice, two protein bands were successfully detected (Fig. 1f, right panel). They corresponded to the sizes of TRPA1a and TRPA1b expressed in HEK293T cells (Fig. 1f, left panel and Supplementary Fig. S2). These two bands were not affected by treatment with a deglycosylation enzyme for 4 hours, indicating that these proteins were not glycosylated and nonglycosylated forms of TRPA1, respectively (Supplementary Fig. S3).

Physical interaction between TRPA1a and TRPAlb. As TRPAla and TRPA1b differed by only 30 amino acid residues, there was no antibody available that could efficiently differentiate between them. Therefore, tagged constructs of TRPAla-EGFP and TRPA1b-FLAG were generated for further analysis. After heterologous expression in HEK293T cells, TRPA1a-EGFP and TRPA1b-FLAG signals were detected using EGFP fluorescence (green) and an anti-FLAG antibody (red), respectively (Fig. 2a). HEK293T cells co-expressing TRPA1a-EGFP with TRPA1bFLAG displayed essentially similar EGFP fluorescence and FLAG immunoreactivity compared with cells expressing either TRPA1aEGFP or TRPA1b-FLAG alone. In addition, TRPA1a-EGFP and TRPA1b-FLAG were highly colocalized on the plasma membrane of HEK293T cells co-expressing the two proteins together (Fig. 2a, Merged). As both TRPAla and TRPA1b were expressed in the same neurons, it was possible that they physically interacted with each other. This possibility was confirmed by coimmunoprecipitation experiments in that there was indeed a physical interaction between TRPA1a and TRPAlb when they were heterologously expressed in HEK293T cells (Fig. 2b). Interestingly, in the native-PAGE analysis, HEK293T cells coexpressing TRPAla-EGFP with TRPAlb-FLAG showed a larger size band (Fig. 2c, white arrowheads, $\sim 920 \mathrm{kDa}$ ) compared with cells expressing TRPAla-EGFP homo-tetramer (Fig. 2c, red arrowhead, $\sim 600 \mathrm{kDa}$ ) or TRPAlb-FLAG homo-tetramer (Fig. 2c, black arrowhead, $\sim 520 \mathrm{kDa}$ ). The native-PAGE result further supported the idea that TRPAla and TRPA1b physically interacted with each other and formed a large complex.

Plasma membrane localization is important for TRPA1 channel function. Thus, interaction between TRPAla and TRPAlb could affect their plasma membrane expression levels. To examine this possibility, a biotinylation assay of plasma membrane proteins was performed. After biotin labelling, plasma membrane proteins were pulled down with avidin-beads and immunoreacted with the appropriate primary antibodies. Both TRPAla and TRPAlb were confirmed to translocate to the plasma membrane (Fig. 2d), with TRPAla plasma membrane levels significantly increased upon co-expression with TRPA1b compared with cells expressing TRPAla alone (Fig. 2e). The plasma membrane expression level of TRPAlb was not changed (Fig. 2f). These results indicated that TRPAla and TRPA1b 
a

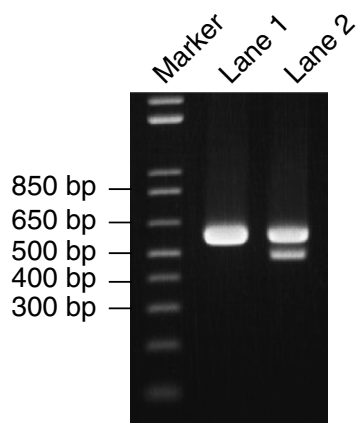

b

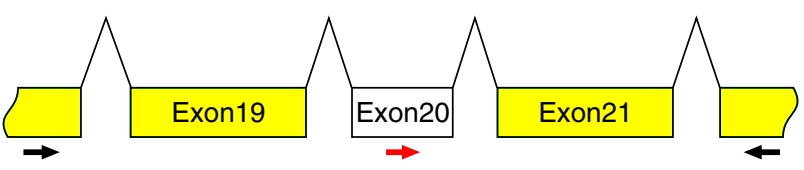

Trpa1a

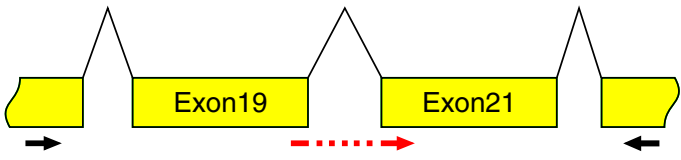

Trpa1b

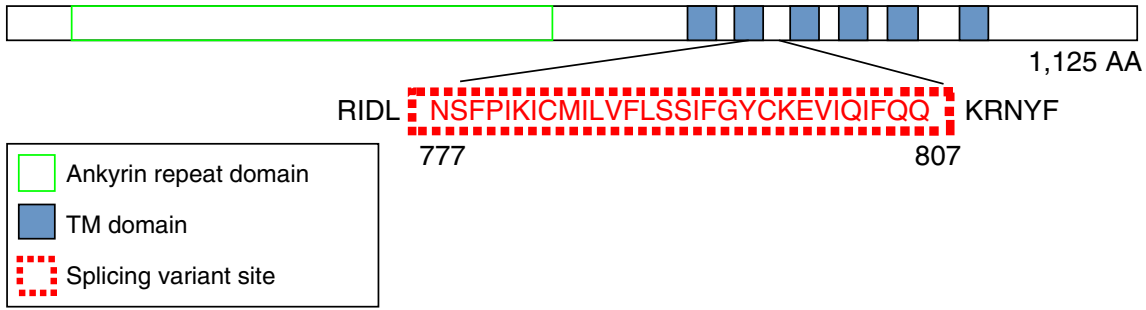

d

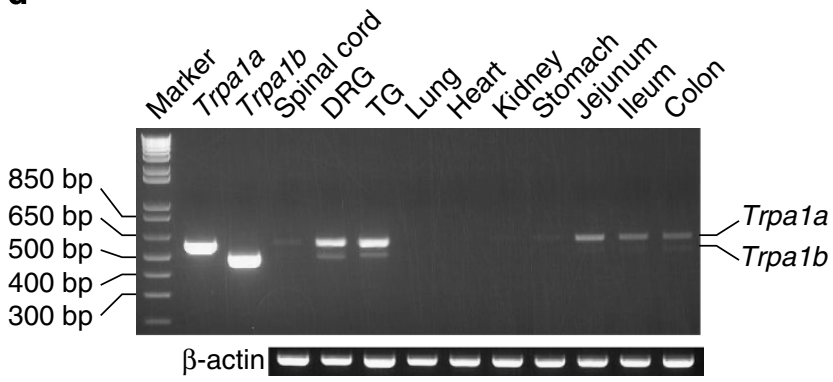

e

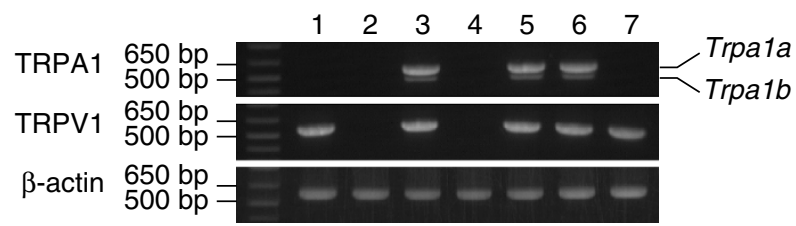

$\mathbf{f}$

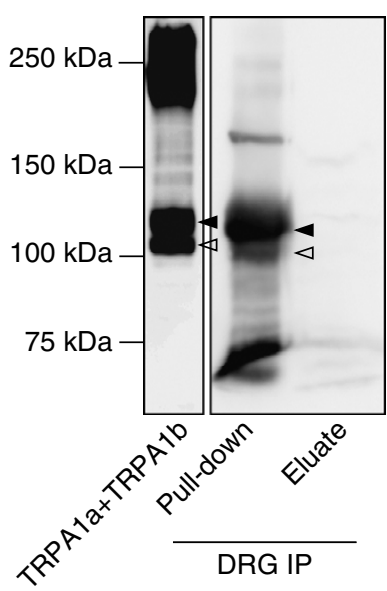

Figure 1 | Identification of an alternative splice variant of the mouse Trpa1 gene from DRG cells. (a) Representative results for RT-PCR using mouse DRG. Lane 1: Trpa1a-positive control; Lane 2: cDNA from mouse DRG. (b) Schematic Trpa1a and Trpa1b mRNA structures. Black arrows indicate the forward and reverse primers for RT-PCR analysis and red arrows indicate forward primers for qRT-PCR analysis. (c) Schematic protein structure of the TRPA1 channel. The dot red box indicates the 30 amino acid residues missing in the TRPA1b protein. The green box indicates the ankyrin repeat domain and the six blue boxes indicate the six predicted transmembrane domains of TRPA1a. (d) Specific expression patterns of Trpa1a and Trpa1b mRNAs were confirmed by RT-PCR analysis of different mouse tissues. (e) Single-cell RT-PCR from mouse DRG neurons. Numbers indicate PCR results from different neurons. Expected sizes of the amplified fragments are $577 \mathrm{bp}, 548 \mathrm{bp}$ and $573 \mathrm{bp}$ for Trpa1, Trpv1 and $\beta$-actin, respectively. (f) Western blotting result after immunoprecipitation of TRPA1 from mouse DRG with an anti-mouse TRPA1 antibody. Black and white arrowheads indicate TRPA1a and TRPA1b proteins, respectively.

physically interacted with each other and that co-expression of TRPAla with TRPA1b enhanced TRPAla expression on the plasma membrane.

TRPA1a and TRPA1b co-expression influences TRPA1 activity. The increased TRPA1 protein expression observed above could lead to enhanced TRPA1 activity. To examine this possibility, electrophysiological experiments using both TRPAla and TRPA1b were performed using a whole-cell patch-clamp method. Mocktransfected HEK293T cells were confirmed not to respond to $100 \mu \mathrm{M}$ allyl isothiocyanate (AITC), whereas cells expressing
TRPAla showed a large inward current. Unexpectedly, cells expressing TRPA1b alone failed to respond to AITC; however, HEK293T cells co-expressing TRPAla with TRPA1b exhibited a larger inward current than that of cells expressing TRPAla alone. The outward-rectifying current-voltage relationships were similar in cells expressing TRPA1a alone and cells expressing TRPA1a with TRPA1b (Fig. 3a).

AITC activates TRPA1 through covalent modification of cysteine residues $^{22,23}$, but TRPA1 can also be activated by different compounds that do not promote covalent modification ${ }^{24-26}$. Three other TRPA1 agonists (2-APB, carvacrol and thymol) that are known to activate TRPA1 through non-covalent modification 
a
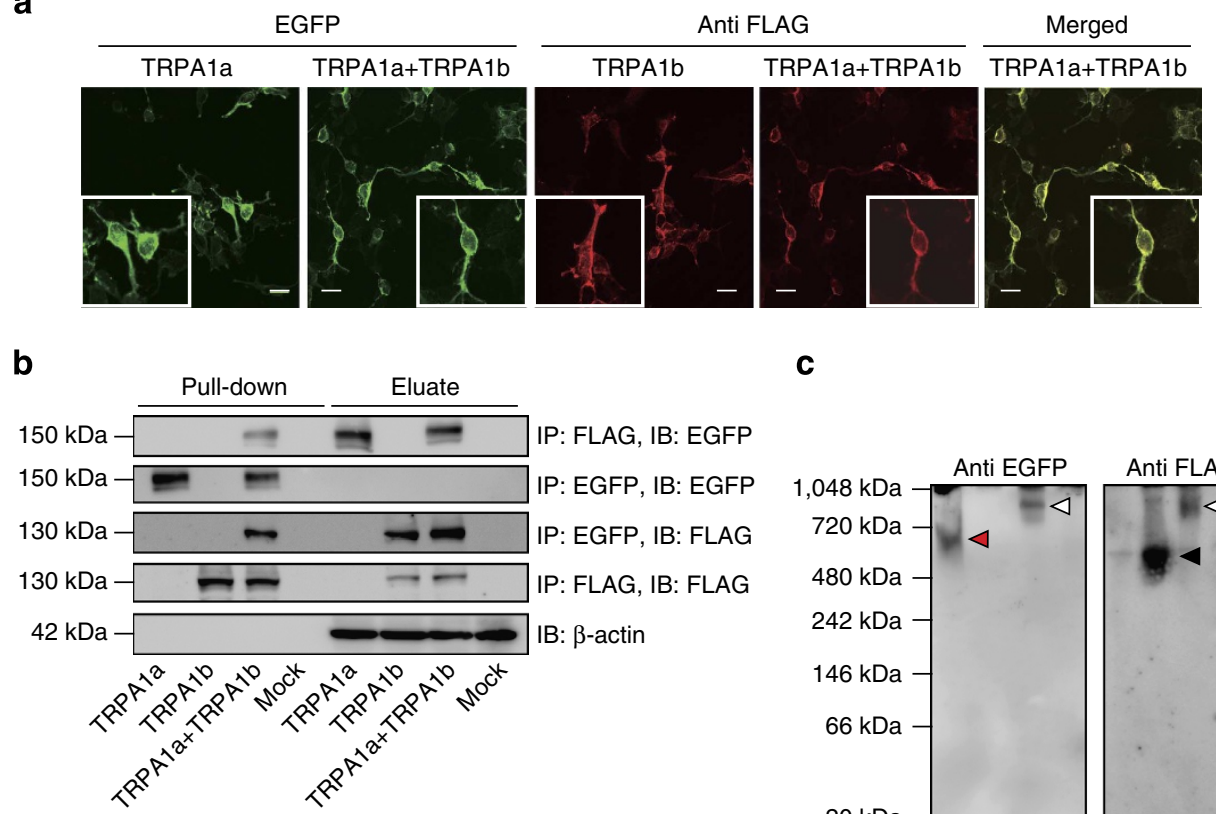

C
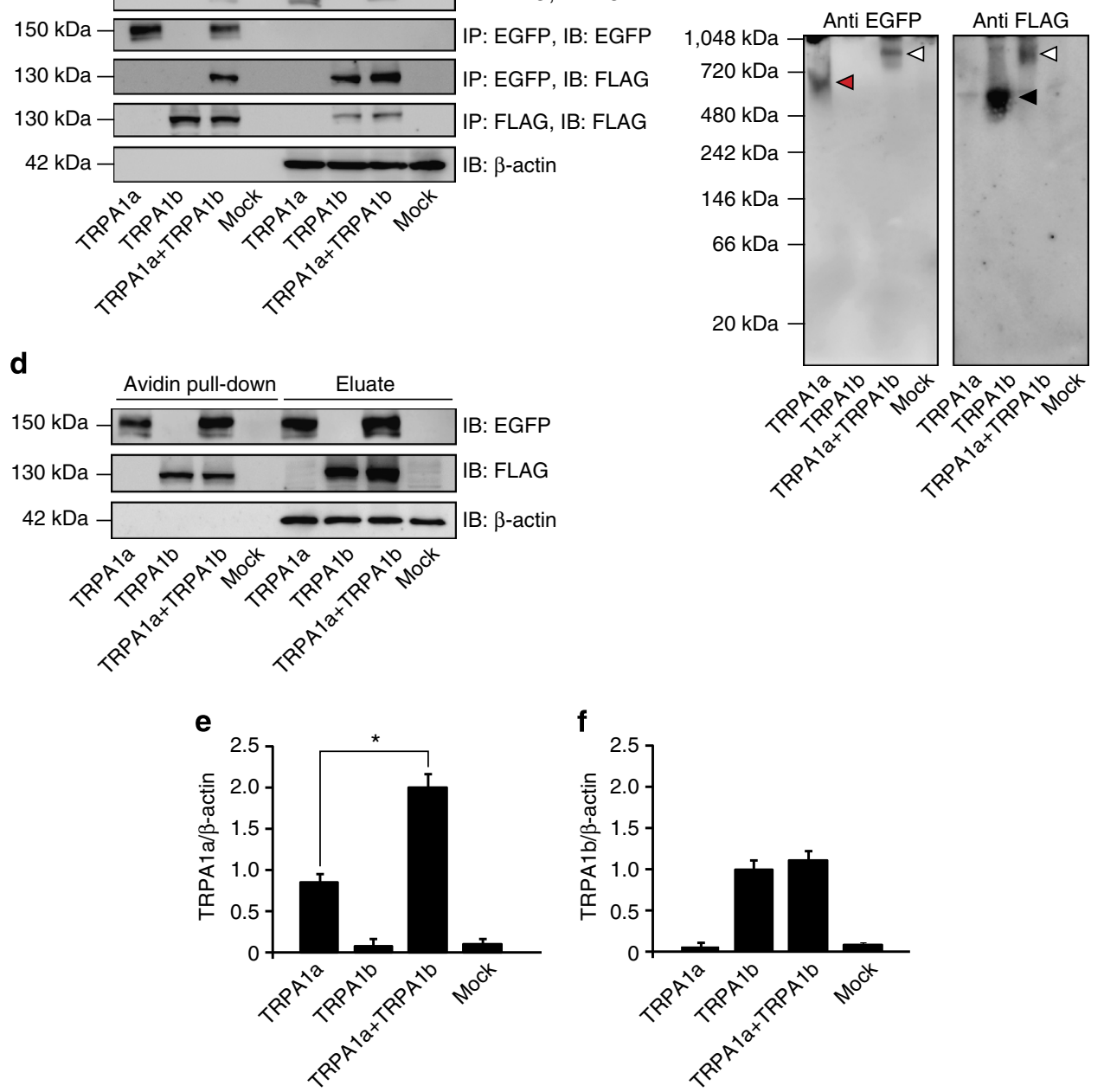

Figure 2 | TRPA1b physically interacts with TRPA1a and regulates its plasma membrane expression. (a) Immunocytochemical analysis of TRPA1a and TRPA1b protein expression in HEK293T cells using TRPA1a-EGFP and TRPA1b-FLAG proteins. Green colour images indicate the cells were stimulated with EGFP fluorescence, whereas red colour images indicate the cells were stained with an anti-FLAG antibody. A merged image (yellow) shows the expression of both TRPA1a-EGFP and TRPA1b-FLAG. Small square boxes indicate the magnified images. Scale bars indicate $20 \mu \mathrm{m}$. (b) Co-immunoprecipitation results of TRPA1a-EGFP and/or TRPA1b-FLAG protein expression in HEK293T cells. Proteins were immunoprecipitated and immunoblotted with either anti-EGFP or anti-FLAG antibody. $\beta$-actin was the loading control for all of the groups. (c) The native-PAGE analysis for TRPA1a-EGFP and TRPA1b-FLAG using an anti-EGFP or anti-FLAG antibody, respectively. Red and black arrowheads possibly indicate TRPA1a and TRPA1b homo-tetramers, respectively. White arrowheads possibly indicate the complex formed by TRPA1a and TRPA1b. (d) Immunoblotting analysis of TRPA1a and TRPA1b protein expression in biotin-labeled plasma membranes. (e,f) Quantitative analysis of the expression of TRPA1a (e) and TRPA1b (f) proteins in the plasma membrane relative to $\beta$-actin. Data represent mean \pm s.e.m. ${ }^{\star} P<0.05, n=5$, one-way ANOVA with Bonferroni's post hoc analysis.

mechanisms were used ${ }^{27}$. As with AITC, none of these agonists induced inward current in HEK293T cells expressing TRPA1b alone, whereas they all induced significantly larger inward currents in HEK293T cells co-expressing TRPAla with TRPA1b compared with cells expressing TRPAla alone (Fig. $3 \mathrm{~b}$ ). These results strongly suggested that, regardless of the activation mechanisms, co- expression of TRPAla with TRPA1b caused significantly larger agonist-induced TRPA1 currents compared with cells expressing TRPAla alone. These significantly increased currents could be easily explained by the biotinylation assay results that showed an increased plasma membrane expression level of TRPAla upon coexpression with TRPA1b in HEK293T cells (Fig. 2). As TRPA1 was 
a

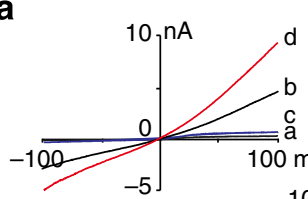

$d$
$b$
$c$
$a$
$\mathrm{mV}$
$100 \mu \mathrm{M}$ AITC
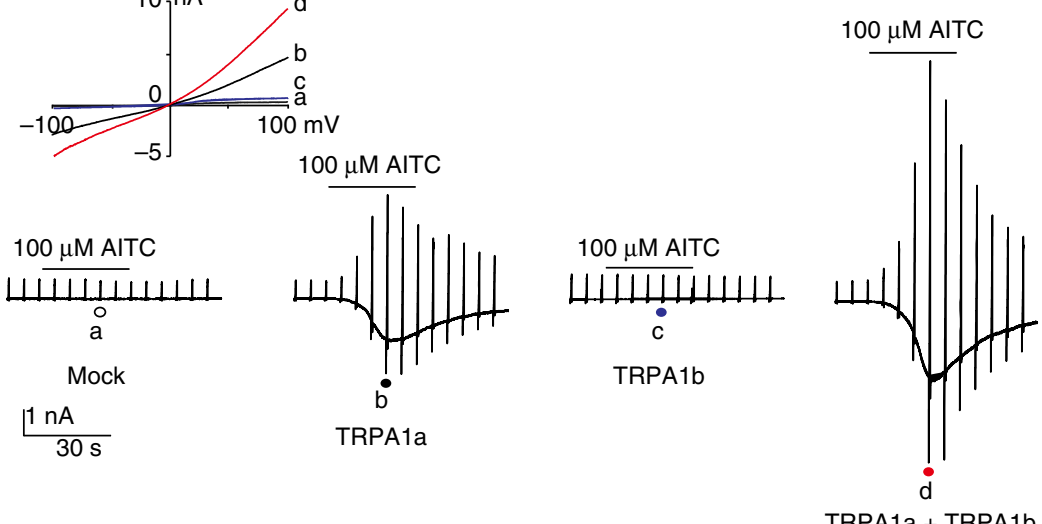

TRPA1b

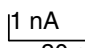

TRPA1a

TRPA1a + TRPA1b

b

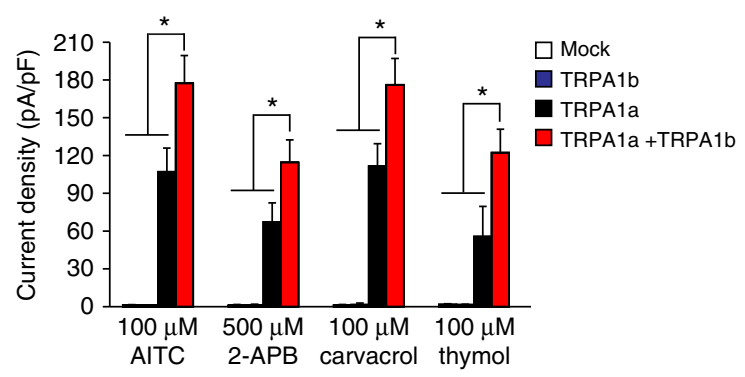

c

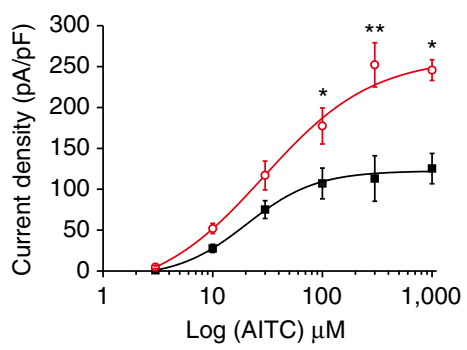

d

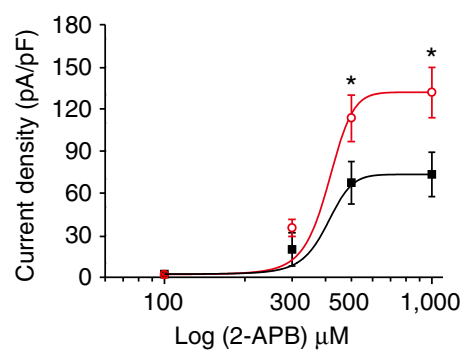

e TRPA1a
TRPA1a + TRPA1b
$\frac{5 \mathrm{pA}}{25 \mathrm{~ms}}$ f

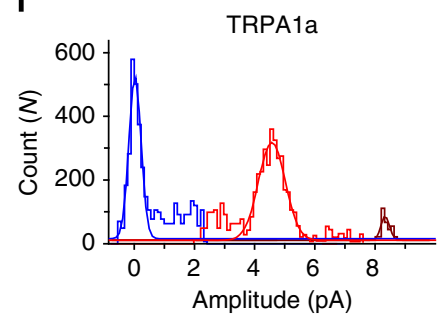

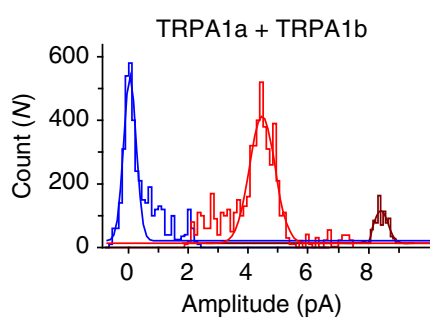

Figure 3 | Co-expression of TRPA1b increases TRPA1a current density in HEK293T cells. (a) Representative whole-cell current traces and currentvoltage $(I-V)$ relationship curves in response to $100 \mu \mathrm{M}$ AITC in control (Mock) cells and cells expressing TRPA1a alone, TRPA1b alone or TRPA1a with TRPA1b. $a, b, c$, and $d$ in the traces indicate the point at which $I-V$ curves were generated. (b) Comparison of TRPA1-mediated current densities activated by four different agonists. Data represent mean \pm s.e.m. ${ }^{\star} P<0.05, n \geq 11$ each, one-way ANOVA with Bonferroni's post hoc analysis. (c,d) Dosedependent curves for AITC-evoked (c) and 2-APB-evoked (d) responses. Red open circles indicate responses in HEK293T cells expressing both TRPA1a and TRPA1b, and black squares indicate responses in cells expressing TRPA1a alone. Data represent mean \pm s.e.m. ${ }^{\star} P<0.05 ;{ }^{\star \star} P<0.01, n \geq 8$ and 9 , respectively, unpaired Student's t-test. (e) Representative $100 \mu \mathrm{M}$ AITC-evoked single-channel current traces from cells expressing TRPA1a alone (upper) and cells expressing TRPA1a and TRPA1b (lower). Holding potential is $-60 \mathrm{mV}$. (f) Open-event histograms of $100 \mu \mathrm{M}$ AITC-evoked single-channel responses from cells expressing TRPA1a alone (left) and cells expressing TRPA1a and TRPA1b (right). Average single-channel conductance was $60.8 \pm 2.6 \mathrm{pS}$ for cells expressing TRPA1a alone and $63.3 \pm 3.0 \mathrm{pS}$ for cells expressing TRPA1a with TRPA1b. TRPA1a $n=20 ;$ TRPA1a + TRPA1b $n=23$.

initially reported as a sensor for noxious cold stimulus ${ }^{3}$, cold response was also examined. However, neither TRPAla, TRPA1b nor TRPAla + TRPAlb was activated by cold stimulus (as low as $10^{\circ} \mathrm{C}$ ) in the absence or presence of $100 \mathrm{nM}$ intracellular calcium, supposedly an enhancer of TRPA1 activity $^{28}$ (Supplementary Fig S4). These results indicated that TRPAla with or without TRPA1b was cold-insensitive. In addition, different transfection ratios of TRPAla and TRPA1b were examined. Even co-expression of 


\begin{tabular}{|c|c|c|}
\hline & $\begin{array}{c}\text { TRPA1a } \\
\text { Mean } \pm \text { s.e.m. }\end{array}$ & $\begin{array}{c}\text { TRPA1a + TRPA1b } \\
\text { Mean } \pm \text { s.e.m. }\end{array}$ \\
\hline \multicolumn{3}{|l|}{ AITC } \\
\hline Hill coefficient & $1.38 \pm 0.12$ & $0.97 \pm 0.21$ \\
\hline $\mathrm{EC}_{50}(\mu \mathrm{M})$ & $20.39 \pm 1.14$ & $29.13 \pm 4.92$ \\
\hline$C D_{\max }(p A / p F)$ & $122.43 \pm 3.69$ & $261.09 \pm 17.1^{\star}$ \\
\hline$C D_{\min }(\mathrm{pA} / \mathrm{pF})$ & $7.75 \pm 1.93$ & $4.45 \pm 0.64$ \\
\hline \multicolumn{3}{|l|}{$2-A P B$} \\
\hline Hill coefficient & $8.93 \pm 0.03$ & $9.02 \pm 0.03$ \\
\hline $\mathrm{EC}_{50}(\mu \mathrm{M})$ & $404.27 \pm 0.16$ & $408.73 \pm 0.15$ \\
\hline$C D_{\max }(p A / p F)$ & $73.88 \pm 0.04$ & $132.94 \pm 0.07^{\star}$ \\
\hline$C D_{\min }(\mathrm{pA} / \mathrm{pF})$ & $2.79 \pm 0.03$ & $3.00 \pm 0.05$ \\
\hline
\end{tabular}

$C D_{\max }$, Maximum current density; $C D_{\min }$, minimum current density.

${ }^{*} P<0.05$ versus TRPA1a, TRPA1a $n=8$; TRPA1a + TRPA1b $n=9$, unpaired Student's $t$-test.

TRPA1a with TRPA1b at a ratio of 2:1 significantly enhanced the current density in response to $300 \mu \mathrm{M}$ AITC, and the enhancement was saturated with a ratio of $1: 1$. This result indicated that TRPA1b interacted with TRPAla in a concentration-dependent manner (Supplementary Fig. S5).

To clarify whether co-expression of TRPAla with TRPA1b influenced the activation properties of TRPAla, the effects of different concentrations of agonists, AITC and 2-APB on TRPA1-mediated currents were examined and dose-dependent curves for AITC- and 2-APB-evoked responses were fitted to Hill equations (Fig. $3 \mathrm{c}, \mathrm{d}$ ). The calculated $\mathrm{EC}_{50}$ values for AITC- and 2-APB-induced activation (Table 1) were quite similar to those stated in a previous report ${ }^{29}$. Although co-expression of TRPAla with TRPA1b did not affect the Hill coefficients or the $\mathrm{EC}_{50}$ values of these two curves compared with TRPAla expression alone, the maximum current density $\left(\mathrm{CD}_{\max }\right)$ in response to both AITC and 2-APB was significantly larger in cells co-expressing TRPAla with TRPA1b compared with cells expressing TRPA1a alone (Table 1 and Fig. 3c,d). These results suggested that coexpression of TRPAla with TRPAlb increased the efficacy but not the potency of AITC and 2-APB actions on TRPA1. This observation was consistent with the two-fold increase in the plasma membrane expression level of TRPAla upon coexpression with TRPA1b (Fig. 2e).

If TRPAla and TRPA1b physically interacted with each other by forming a heteromer, the single-channel properties could be affected. To examine this possibility, inside-out single-channel recordings were performed in HEK293T cells expressing TRPA1a alone and in cells co-expressing TRPAla with TRPA1b (Fig. 3e). Surprisingly, TRPA1a and TRPA1b co-expression did not affect open-time kinetics (Supplementary Fig. S6) or single-channel conductance (Table 2). However, $\mathrm{NP}_{\mathrm{o}}$ values from cells expressing TRPAla with TRPAlb in response to $100 \mu \mathrm{M}$ AITC were significantly higher than those from cells expressing TRPAla alone (Fig. $3 \mathrm{f}$ and Table 2). These results could be explained by an increase in channel number in the patch membrane. These data suggested that the increase in agonist responses was possibly due to the enhanced expression levels of TRPAla by co-expression with TRPAlb, but not changing the single-channel conductance.

TRPA1b interacts and regulates TRPA1a in DRG neurons. All studies thus far had been performed using a heterologous expression system with HEK293T cells. Next, mouse DRG neurons were chosen to confirm the TRPAla and TRPAlb interaction observed in HEK293T cells. Trpalb gene was
Table 2 | Single-channel recordings from TRP-expressing HEK293T cells.

\begin{tabular}{lcc} 
& $\begin{array}{c}\text { TRPA1a } \\
\text { Mean } \pm \text { s.e.m. }\end{array}$ & $\begin{array}{c}\text { TRPA1a }+ \text { TRPA1b } \\
\text { Mean } \pm \text { s.e.m. }\end{array}$ \\
\hline AlTC & & \\
Conductance $(\mathrm{pS})$ & $60.83 \pm 2.59$ & $63.33 \pm 2.98$ \\
NP & $0.45 \pm 0.13$ & $0.77 \pm 0.08^{\star}$ \\
Tau-1 & $22.47 \pm 6.02$ & $20.91 \pm 3.38$ \\
Tau-2 & $2.50 \pm 0.36$ & $1.77 \pm 0.17$ \\
\hline${ }^{*}<0.05$ versus TRPA1a, TRPA1a $n=20 ;$ TRPA1a + TRPA1b $n=23$, unpaired Student's $t$-test. \\
\hline
\end{tabular}

overexpressed in WT DRG neurons by electroporation. WT DRG neurons treated with vector pcDNA3.1 served as Control. The changes in fura- 2 ratio corresponding to cytosolic calcium concentrations $\left(\left[\mathrm{Ca}^{2+}\right]_{\mathrm{i}}\right)$ of the DRG neurons were recorded in response to AITC $(100 \mu \mathrm{M})$ and capsaicin (Cap, $0.5 \mu \mathrm{M})$ (Fig. 4a). The responses were normalized to those of ionomycin and statistically analysed. The data indicated that AITC-, but not Cap-evoked ratio increases were significantly larger in TRPA1boverexpressing neurons compared with that in control neurons (Fig. 4b). As previous reports showed that DRG neurons have distinct TRP channel expression patterns ${ }^{2,8,30}$, cell populations were also compared between Control and TRPAlb overexpressing DRG neurons. Overexpression of TRPA1b did not significantly influence endogenous TRP channel expression patterns (Fig. 4c), indicating that TRPA1b only affected TRPA1a but not the TRPV1 channel. In addition, changes in real $\left[\mathrm{Ca}^{2+}\right]_{i}$ in response to AITC were calculated from these two groups using a standard curve. The results showed that mouse DRG neurons expressing exogenous TRPA1b had a significantly larger increase in $\left[\mathrm{Ca}^{2+}\right]_{\mathrm{i}}$ (Supplementary Fig. S7), similar to the results calculated from the normalized ratio.

TRPA1KO DRG neurons were also used to further examine the effects of TRPAla and TRPA1b. TRPA1KO DRG neurons were electroporated with either control (pcDNA3.1), TRPA1a alone or TRPAla with TRPA1b plasmid DNAs. Expression of TRPA1a alone and TRPAla with TRPAlb rescued responses to AITC in the TRPA1KO neurons, whereas control cells showed no response (Fig. 5a). Interestingly, we observed a significantly larger ratio change in neurons co-expressing TRPA1a with TRPAlb compared with those expressing TRPAla alone (Fig. 5b), similar to the experiment performed with WT neurons (Fig. $4 \mathrm{~b}$ and Supplementary Fig. S7). Taken together, these results suggested that TRPA1b not only regulated TRPA1a expression in the heterologous expression system with HEK293T cells but also regulated TRPA1a expression in native sensory neurons.

Changes of Trpala and Trpalb mRNAs in two animal models. Recent studies suggested that activation of TRPA1 contributes to mechanical hyperalgesia under inflammatory pain conditions ${ }^{31,32}$, where Trpa1 mRNA was found to increase rapidly and then return to basal level ${ }^{8,33}$. However, the regulatory mechanism of TRPA1 involvement in inflammatory pain conditions has remained unclear. Therefore, a complete Freund's adjuvant (CFA)-induced inflammatory pain model was used to explore whether TRPA1b modulated TRPA1a under pathological pain conditions. In WT mice, intraplantar injection of CFA into the hind paw induced mechanical hyperalgesia that was confirmed by the von Frey filament test. The reduction lasted for 14 days and was not observed in the control mice injected with saline. Intraplantar injection of CFA into TRPA1KO mice hind paws, however, significantly reduced the ipsilateral 50\% mechanical 

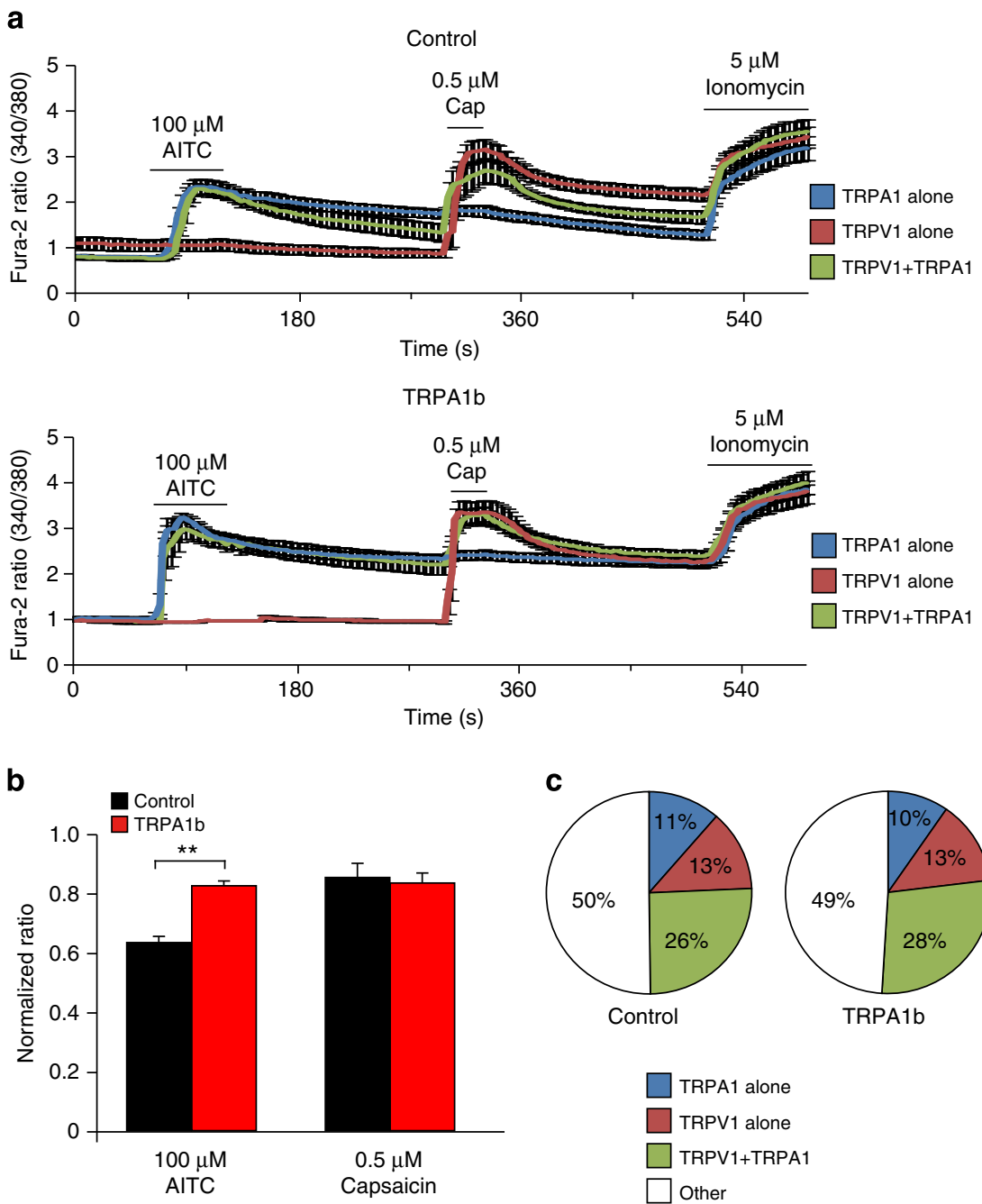

Figure 4 | TRPA1b overexpression in WT DRG cells enhanced the AITC- but not the capsaicin-evoked increase in the fura-2 ratio. (a) Changes in the fura- 2 ratio in vector-introduced (Control, upper) and TRPA1b-introduced (lower) DRG neurons from WT mice. Black bars indicate agonist application periods. Responses to TRPA1 alone (blue), TRPV1 alone (red), and TRPA1 with TRPV1 (green) are shown. (b) AITC and Cap responses (normalized to the ionomycin responses) were compared. Data represent mean \pm s.e.m. ${ }^{\star \star} P<0.01$, Control $n=343$; TRPA1b $n=308$, unpaired Student's t-test. (c) Summary of AITC- and Cap-positive cell populations from vector-introduced (Control, left) and TRPA1b-introduced (right) DRG neurons.

threshold only on days 1 and 2 (Fig. 6a). These findings indicate that TRPA1 function is of greater importance in the later stage of inflammation. No significant changes in the $50 \%$ mechanical threshold of the contralateral hind paws were observed among the three groups tested (Supplementary Fig. S8a). The expression levels of Trpa1 mRNA in mouse DRG neurons from lumbar 4, 5 and 6 sections, which correspond to the affected hind paw neurons, were evaluated by $\mathrm{qRT}-\mathrm{PCR}$ analysis at different time points after the injection. The expression level of Trpala mRNA from ipsilateral DRG neurons of CFA-injected mice was significantly increased on day 1 compared with that from ipsilateral DRG neurons of saline-injected mice (Fig. 6c). The increased Trpala expression level returned to basal level on day 5 after injection, which is consistent with the previous studies ${ }^{8,33}$. On the other hand, the expression level of Trpalb mRNA gradually but significantly increased for 14 days after injection (Fig. 6d). No significant difference was observed in either Trpala or Trpalb mRNA expression level from contralateral DRG cells of these groups (Supplementary Fig. S8c). Functional analysis of TRPA1 was examined by using a calcium-imaging method (Fig. 6g). Consistent with the behavioural results, AITC responses in ipsilateral DRG neurons from CFA-injected WT mice were significantly larger compared with those in DRG neurons from saline-injected WT mice. DRG neurons from TRPA1KO mice did not respond to AITC. Furthermore, contralateral DRG neurons did not exhibit significant differences in AITC responses between saline- and CFA-injected mice (Supplementary Fig. S8e).

Some research has demonstrated that TRPA1 has an important role in neuropathic pain ${ }^{8,34-36}$, whereas other studies did not support that conclusion ${ }^{37-39}$. In this study, a partial sciatic nerve ligation (PSL)-induced neuropathic pain model was selected to examine the involvement of TRPA1 and to determine the mRNA expression levels of Trpala and Trpa1b. The same experimental time course paradigm was applied to WT sham-operated, WT PSL-operated and TRPA1KO PSL-operated mice as in the CFAinduced inflammatory pain model. The 50\% mechanical threshold was significantly reduced in PSL-operated WT mice 1 day after ligation compared with sham-operated WT mice. The reduced mechanical threshold gradually reached a plateau on day 14 after ligation. Interestingly, PSL-operated TRPA1KO mice exhibited only a partial reduction in the 50\% mechanical threshold (Fig. 6b), which suggested that TRPA1 could be involved in PSL-induced mechanical hypersensitivity to some extent. No significant changes in the $50 \%$ mechanical threshold of 
a
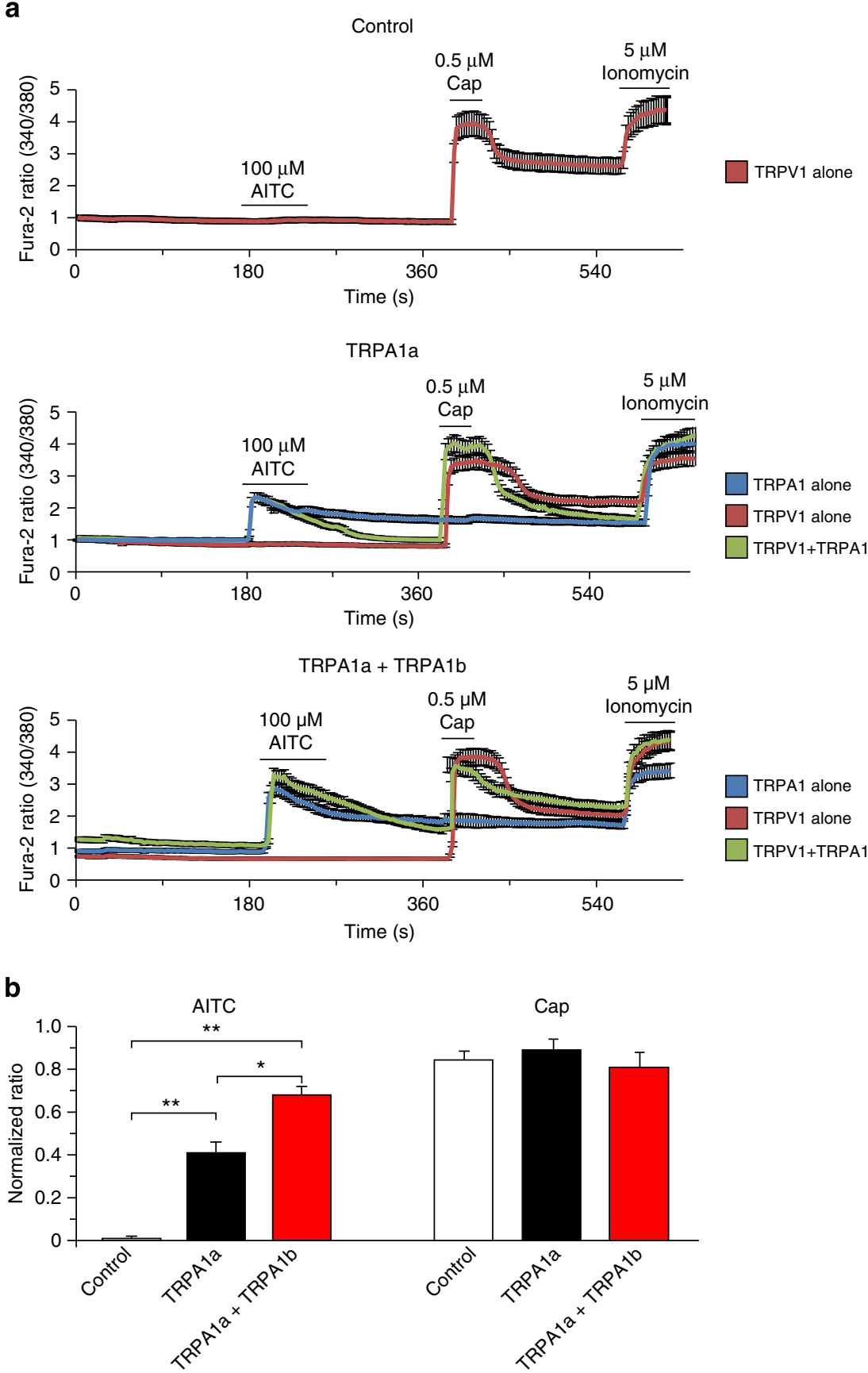

Figure 5 | Exogenous expression of TRPA1a and TRPA1b rescued the AITC-evoked increase in the fura-2 ratio in TRPA1KO DRG cells. (a) Changes in the fura-2 ratio in vector-introduced (Control, upper), TRPA1a-introduced (middle) and TRPA1a with TRPA1b-introduced (lower) DRG neurons from TRPA1KO mice. Black bars indicate agonist application periods. Responses to TRPA1 alone (blue), TRPV1 alone (red) and TRPV1 with TRPA1 (green) are shown. (b) AITC responses (normalized to the ionomycin responses) were compared. Data represent mean \pm s.e.m. ${ }^{\star} P<0.05$, ${ }^{\star \star} P<0.01$, Control $n=298$; TRPA1a $n=312$; TRPA1a + TRPA1b $n=298$, one-way ANOVA with Bonferroni's post hoc analysis.

the contralateral hind paws were observed in the three groups tested (Supplementary Fig. S8b). In ipsilateral DRG from PSLoperated WT mice, the Trpala mRNA expression level significantly increased on day 5 after ligation and returned to the basal level on day 14 (Fig. 6e). On the other hand, the Trpalb mRNA expression level gradually increased for 14 days after ligation (Fig. 6f). However, neither the Trpala nor Trpalb mRNA expression level was significantly changed in contralateral DRG neurons in PSL-operated or sham-operated mice (Supplementary Fig. S8d). Similar to the results obtained with the CFA model, AITC responses in ipsilateral DRG neurons from PSL-operated
WT mice were significantly larger compared with those in DRG neurons from sham-operated WT mice (Fig. 6g). DRG neurons from TRPA1KO mice did not respond to AITC. In addition, contralateral DRG did not exhibit significant differences in AITC responses between sham- and PSL-operated mice (Supplementary Fig. S8e). Therefore, the mRNA expression levels and functional analysis results indicated that TRPA1a is involved in both pain models (CFA-induced inflammatory pain and PSL-induced neuropathic pain models). These data indicate that TRPA1a could be regulated by its alternative splice variant TRPA1b in the late stages of these conditions. 
a

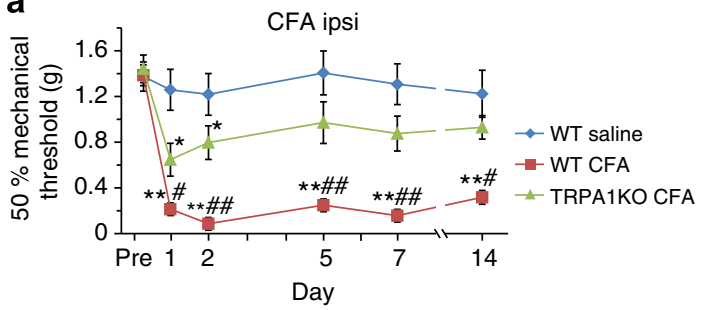

b

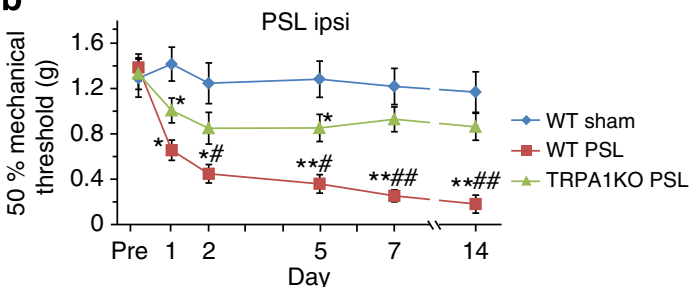

$\mathbf{g}$

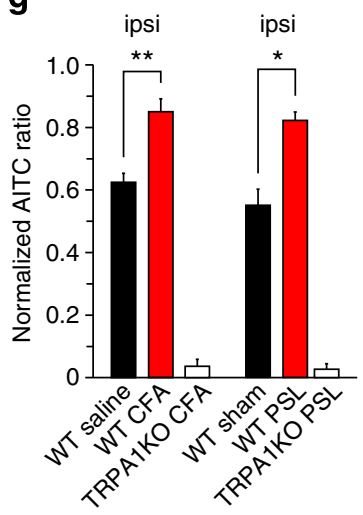

C

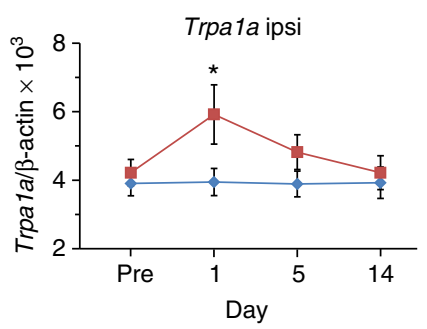

e

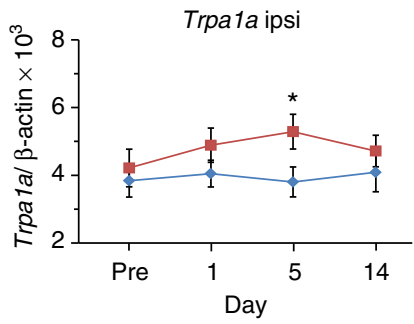

d

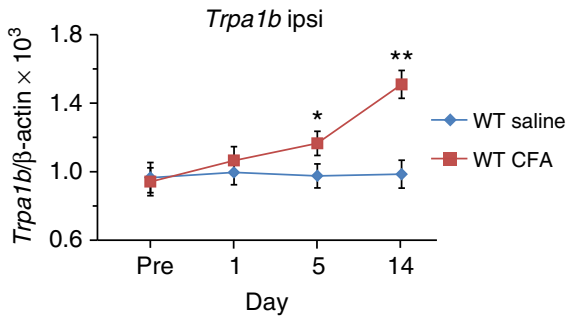

$\mathbf{f}$

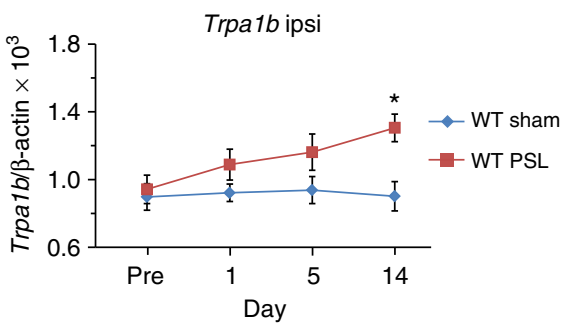

Figure 6 | Behavioural analysis and mRNA expression patterns of Trpa1a and Trpa1b in two pain models. (a,b) Changes in the ipsilateral $50 \%$ mechanical thresholds of CFA-induced inflammatory pain (a) and PSL-induced neuropathic pain (b) models. Data represent mean \pm s.e.m. ${ }^{\star} P<0.05$, ${ }^{\star \star} P<0.01$ versus WT saline or sham, $\# P<0.05$, \#\#P<0.01 versus TRPA1KO CFA or PSL, WT saline (blue) $n=36 ;$ WT CFA (red) $n=48$; TRPA1KO CFA (green) $n=5$; WT sham (blue) $n=40$; WT PSL (red) $n=44$; TRPA1KO PSL (green) $n=5$, one-way ANOVA with Bonferroni's post hoc analysis. (c,d) Time courses of Trpa1a (c) and Trpa1b (d) mRNA expression levels in ipsilateral DRG from CFA-induced inflammatory pain model. Data represent mean \pm s.e.m. ${ }^{\star} P<0.05,{ }^{\star \star} P<0.01$ versus WT saline, WT saline $n=9$; WT CFA $n=12$, unpaired Student's $t$-test. (e,f) Time courses of Trpa1a (e) and Trpa1b (f) mRNA expression levels in ipsilateral DRG from PSL-induced neuropathic pain model. Data represent mean \pm s.e.m. ${ }^{\star} P<0.05$, ${ }^{\star \star} P<0.01$ versus WT sham, WT sham $n=10$; WT PSL $n=11$, unpaired Student's t-test. (g) Changes in the fura-2 ratio in ipsilateral DRG 2 weeks after CFA-injection and PSL-operation. Saline-injected and sham-operated WT mice (black), CFA-injected and PSL-operated WT mice (red), CFA-injected and PSL-operated TRPA1KO mice (white). Data represent mean \pm s.e.m. ${ }^{\star} P<0.05,{ }^{\star}{ }^{\star} P<0.01$ versus WT saline or sham, WT saline $n=275 ;$ WT CFA $n=286$; TRPA1KO CFA $n=284$; WT sham $n=239$; WT PSL $n=275$; TRPA1KO PSL $n=300$, one-way ANOVA with Bonferroni's post hoc analysis.

\section{Discussion}

TRPA1 is reportedly expressed in a subpopulation of peptidergic sensory neurons that also co-express TRPV1 (ref. 3). It can be activated in response to many chemicals, natural plant extracts and a large number of pro-algesic agents ${ }^{40,41}$. Activation of TRPA1 in sensory neurons causes pain-related behaviours in mice $^{3}$, whereas TRPA1KO mice are insensitive to TRPA1 agonists and display impaired pain-related behaviours in response to these compounds ${ }^{21}$. TRPA1KO mice also did not develop neurogenic inflammation and hyperalgesia compared with WT animals ${ }^{34,42}$. These results suggest that TRPA1 is not only the primary receptor for these compounds in nociceptive neurons but also is involved in inflammatory and neuropathic pain diseases where mechanical hyperalgesia is observed. However, the mechanisms for the latter phenomena are not completely understood.

In the present study, we identified a mouse-specific alternative splice isoform of the TRPA1 channel, TRPA1b, which lost part of TM2 and the adjacent intracellular loop. The loss of these 30 amino acids would seem likely to create a non-functional channel. This would explain why TRPA1b alone has no activity and would also be consistent with TRPA1b having no effect on the singlechannel properties of TRPAla currents. These results might suggest that TRPA1b functions as a dominant-negative isoform. However, it was not the case, and instead TRPA1b seems to make TRPAla more active or at least more prevalent on the cell 
surface. Moreover, we observed that dynamic changes in mRNA expression levels of Trpala and Trpalb occurred under inflammatory and neuropathic pain conditions. Trpala and Trpa $1 b$ mRNAs were found to co-exist in the same DRG neurons. Co-immunoprecipitation confirmed that TRPAla and TRPA1b physically interacted with each other. Interestingly, the membrane expression level of TRPAla significantly increased upon co-expression with TRPA1b. Although the detailed mechanism remains unclear, it is possible that translocation of TRPAla could be enhanced by physical interaction with TRPA1b. Alternatively, it is possible that the stability or rate of internalization of the complex could be affected by TRPA1b, which would be analogous to the findings of several studies showing that splice variants have different internalization or post-endocytic sorting fates ${ }^{43,44}$.

The native-PAGE results indicated that a large-sized complex could be formed by TRPAla and TRPA1b compared with that of TRPAla and TRPAlb homo-tetramers. According to the apparent band size of the complex, the complex might be formed in a 1:1 ratio of TRPA1a and TRPA1b, namely four TRPA1a subunits and four TRPA1b subunits. Whole-cell patch-clamp recording data are consistent with the biochemical data in that co-expression of TRPAla with TRPA1b enhanced TRPA1 activity regardless of activation mechanism, proving functional interaction between TRPA1a and TRPA1b. Surprisingly, single-channel unitary conductance and the open-time kinetics of the complex remained the same as that of TRPAla homo-tetramer, which indicates that a heteromer of TRPAla with TRPA1b is less possible.

Besides the increased expression level and channel activity of TRPA1a upon co-expression with TRPA1b in HEK293T cells, we also demonstrated that overexpression of TRPA1b in primary DRG neurons increased AITC-, but not capsaicin-evoked $\left[\mathrm{Ca}^{2+}\right]_{\mathrm{i}}$ responses. Those results indicated that TRPAlb selectively interacted with and regulated TRPA1a, but not TRPV1 in sensory neurons, although there are several reports regarding functional and/or physical interaction between TRPV1 and TRPA1 (ref. 45). These results indicated that TRPAlb regulated TRPAla activity not only in a heterologous expression system but also in native sensory neurons by a mechanism that increased plasma membrane TRPA1a expression level.

Even though TRPAlb interacts with and regulates TRPAla, the relatively low expression level of Trpalb mRNA compared with Trpala under physiological conditions could restrict its physiological significance. However, it is possible that mRNA expression patterns and levels of Trpa1a or Trpa1b could be changed under certain pathological conditions. Indeed, analysis of two wellestablished mouse pain models (CFA-induced inflammatory pain and PSL-induced neuropathic pain models) confirmed this hypothesis. Both expression patterns of Trpala and Trpalb mRNAs changed dynamically in these models. Similar to the previous studies ${ }^{8,33}$, Trpala mRNA increased in the early stage of inflammatory pain and returned to the basal level. In contrast, the Trpalb mRNA level increased slowly for 2 weeks after CFA injection or PSL operation. The fact that the response of TRPA1a to AITC was enhanced when TRPAla and TRPA1b were coexpressed at a ratio of 2:1 suggests that a small increase in TRPA1b in pathological conditions possibly enhances TRPA1a expression, leading to the increased TRPA1 activity. These interesting results could explain how TRPA1 expression contributed to mechanical hyperalgesia with such rapid rates of increase and decrease. According to our study, increased Trpala expression in the early inflammation stage is sufficient to contribute to mechanical hyperalgesia, but in later stages when Trpala expression has returned to basal level, the slowly but significantly increased amount of Trpalb could maintain TRPA1a expression at a level that could sustain mechanical hyperalgesia.
Similar to the inflammatory pain model, we observed a rapid and transient increase in Trpala expression and slow and sustained increase in Trpalb in the PSL-induced neuropathic pain model. This sustained increase in Trpalb could be involved in the prolonged mechanical hyperalgesia in the neuropathic pain model as also observed in the inflammatory pain model.

Alternative splicing is a well-characterized enzyme-mediated process that is precisely regulated by several critical proteins ${ }^{46}$. Interestingly, recent studies indicated that there is a relationship between different kinds of diseases and alternative splicing ${ }^{47,48}$. The best-studied splice regulators are members of the SR (serine and arginine) proteins and hnRNP families. If the enzymatic activities of these proteins were affected during some pathologic states, such as inflammation, it is easy to imagine that alternative splicing would also be changed and diversify the products from the pre-mRNAs. However, the mechanistic relationships between various kinds of diseases and alternative splicing remain largely unknown. Recently, much progress has been achieved concerning the relationship between inflammatory disease and alternative splicing. Inflammatory factors, such as cytokines, are believed to be capable of altering pre-mRNA alternative splicing in pancreatic $\beta$-cells ${ }^{49}$. Similar relationships have also been described in many other reports ${ }^{48,50,51}$. The relation between changes in Trpalb and inflammation could be explained in a similar way although the precise mechanisms for the dynamic changes in Trpala and Trpalb expression patterns remain unclear.

In summary, this study has identified a mouse TRPA1 alternative splice variant, TRPAlb, which interacts with TRPAla. Furthermore, dynamic changes are observed in Trpala and Trpalb expression patterns under CFA-induced inflammatory and PSL-induced neuropathic pain conditions. These results could provide new insights for understanding the relationship between diseases and alternative splicing of ion channels.

\section{Methods}

Animals. Male C57Bl/6NCr mice (4-6-week old) and SD rats (3-week old) were housed in a controlled environment $\left(12 \mathrm{~h}\right.$ light/dark cycle; $25^{\circ} \mathrm{C} ; 50-60 \%$

humidity) with food and water ad libitum. For the inflammatory pain model, $20 \mu \mathrm{l}$ CFA was administered to the left hind paw. For the neuropathic pain model, the mice were anesthetized and the right sciatic nerve was exposed. Part of the sciatic nerve was transected with a thread. Saline-injected and sham-operated mice served as controls. The $50 \%$ mechanical thresholds were determined with a von Frey test by an up-down method of Dixon ${ }^{52}$ on days 1, 2, 5, 7 and 14. The mice were killed after 1, 5, 7 and 14 days for qRT-PCR, and for calcium-imaging experiments after 14 days. All procedures involving the care and use of animals were approved by the Institutional Animal Care and Use Committee of the National Institutes of Natural Sciences and carried out in accordance with the National Institutes of Health Guide for the care and use of laboratory animals (NIH publication No. 85-23, revised 1985). TRPA1 knockout mice were generously provided by Dr David Julius (UCSF, San Francisco, California, USA).

Molecular cloning of TRPA1a and TRPA1b. Trpa1a and Trpa1b were cloned into the pcDNA3.1 vector using KOD FX DNA polymerase. Forward and reverse primers were $5^{\prime}$-AAGGATCCGCCACCATGAAGCGCGGC- $3^{\prime}$ and $5^{\prime}$-GAGC GGCCGCCTAAAAGTCCGGGTGG-3' respectively. For tagged vector construction, forward and reverse primers were $5^{\prime}$-AAGCTAGCGCCACCATGAAGCGC GGC- $3^{\prime}$ and $5^{\prime}$-AAGGTACCGCAAAGTCCGGGTGG- $3^{\prime}$ for Trpala, $5^{\prime}$-AAGGAT CCGCCACCATGAAGCGCGGC- $3^{\prime}$ and $5^{\prime}$-AAGCGGCCGCAAAGTCCGGG TGG-3' for Trpa1b. After Trpa1a was digested with NheI and KpnI and Trpa1b with BamHI and NotI, they were subcloned into pEGFP-N1 and pcDNA-FLAG vectors, respectively.

RT-PCR and single-cell RT-PCR. C57Bl/6NCr mouse and SD rat DRG were rapidly collected and total RNA was isolated. Human DRG total RNA was purchased from Clontech Laboratories Incorporation. RT-PCR was performed using the SuperScript III kit from Life Technologies. For single-cell RT-PCR, a glass pipette was used to aspirate cytosolic components from DRG neurons after making a whole-cell configuration. RT-PCR was performed in the same way as above. RT-PCR products were purified by ethanol precipitation and the pellets were used for the second PCR by using the same primers. Forward and reverse primers were $5^{\prime}$-AACTCCTCAACCACCCTGTG- $3^{\prime}$ and $5^{\prime}$-CTGAGGCCAA 
AAGCCAGTAG- $3^{\prime}$ for mouse Trpa1, 5'-AACTCCACCCCACACTGAAG- $3^{\prime}$ and $5^{\prime}$-TCGCCTCTGCAGGAAATACT- $3^{\prime}$ for mouse $\operatorname{Tr} p v 1,5^{\prime}$-GAGCTCCTCAAC CACCCTGTG- $3^{\prime}$ and $5^{\prime}$-GCTGAGGCCAAAAGCCAATAG- $3^{\prime}$ for rat Trpal, $5^{\prime}$-GAGCTTCTCAATCATCCTGTG- $3^{\prime}$ and $5^{\prime}$-GCTGAGTCCAAAAGCCAG AAGA-3' for human TRPA1, 5'-TGTTACCAACTGGGACGACA-3' and $5^{\prime}$-AAGGAAGGCTGGAAAAGAGC- $3^{\prime}$ for $\beta$-actin.

qRT-PCR. Trpala and Trpalb mRNA copy numbers were determined by SYBR Green. Forward and reverse primers were $5^{\prime}$-CAAGTATATTTGGATATTGCAAA GAAG- $3^{\prime}$ and $5^{\prime}$-CTGAGGCCAAAAGCCAGTAG- $3^{\prime}$ for Trpal $a, 5^{\prime}$-GAATAGA CACTCTGAAAAGGAATTAC- $3^{\prime}$ and $5^{\prime}$-CTGAGGCCAAAAGCCAGTAG- $3^{\prime}$ for Trpal $b, 5^{\prime}$-GACAGGATGCAGAAGGA- $3^{\prime}$ and $5^{\prime}$-TGATCCACATCTGCTG GAA- $3^{\prime}$ for $\beta$-actin. Data were analysed using ABI-7700 SDS software.

Maintenance of HEK293T cells and immunocytochemistry. Human embryonic kidney-derived 293T (HEK293T) cells were maintained in DMEM containing $10 \%$ heat-inactivated FBS, 100 units $^{-1}$ penicillin and streptomycin and $2 \mathrm{mM}$ L-glutamine at $37^{\circ} \mathrm{C}$ and $5 \% \mathrm{CO}_{2}$. HEK293T cells were transfected with the appropriate plasmids using Lipofectamine reagent from Life Technologies. TRPAla alone group was transfected with $0.1 \mu \mathrm{g}$ Trpala and $0.9 \mu \mathrm{g}$ empty pcDNA3.1 plasmid. TRPA1b alone group was transfected with $0.9 \mu \mathrm{g}$ Trpalb and $0.1 \mu \mathrm{g}$ empty pcDNA3.1 plasmid. TRPAla + TRPAlb group was transfected with $0.1 \mu \mathrm{g}$ Trpala and $0.9 \mu \mathrm{g}$ Trpalb. After incubation for $18 \mathrm{~h}$, cells were washed two times with PBS and fixed using 4\% PFA. Cells were then washed twice with PBS and once with PBS-T, and blocked for $1 \mathrm{~h}$ at room temperature. Cells were incubated with an anti-FLAG antibody (Sigma-Aldrich) diluted 1:1,000, for $1 \mathrm{~h}$ at room temperature followed by three washes with PBS-T. Anti-mouse Alexa Fluor Dyes 594 (Molecular Probes) was incubated with cells for $1 \mathrm{~h}$ at room temperature. After three washes with PBS and three washes with MilliQ water, cells were mounted onto glass slides.

Co-immunoprecipitation of TRPA1a and TRPA1b. HEK293T cells were transfected using the same transfection ratio and protocol as in the immunocytochemistry experiment. Cells were lysed in $100 \mu \mathrm{l}$ RIPA lysis buffer with protease inhibitor cocktail. Cell lysates were pre-cleared with protein G Sepharose beads with rotation at $4{ }^{\circ} \mathrm{C}$ for $1 \mathrm{~h}$. The supernatants were collected after centrifugation and incubated with $1 \mu \mathrm{l}$ anti-FLAG (Sigma-Aldrich) or $1 \mu \mathrm{l}$ anti-EGFP (Wako Pure Chemical Industries) antibody overnight at $4{ }^{\circ} \mathrm{C}$. Samples were precipitated with $20 \mu \mathrm{l}$ protein G Sepharose beads for $1 \mathrm{~h}$. Samples were denatured at $95^{\circ} \mathrm{C}$ for $5 \mathrm{~min}$, separated on a $6 \%$ SDS-PAGE gel and transferred onto a PVDF membrane. The membrane was blocked overnight at $4{ }^{\circ} \mathrm{C}$ and then incubated with an anti-EGFP antibody or anti-FLAG antibody, each diluted 1:1,000, for $1 \mathrm{~h}$ at room temperature. After three washes with PBS-T, the membrane was incubated for $1 \mathrm{~h}$ at room temperature with an anti-mouse IgG HRP-linked antibody (Cell Signaling Technology) diluted 1:5,000.

DRG immunoprecipitation. DRG cells were rapidly isolated from 10 mice and lysed in $500 \mu \mathrm{l}$ RIPA buffer with protease inhibitors. Half amount of protein was treated with a deglycosylation enzyme (New England Biolabs) at $37^{\circ} \mathrm{C}$ for $4 \mathrm{~h}$. Protein was added to $10 \mu \mathrm{l}$ rabbit anti-mouse TRPA1 polyclonal antibody (generously provided by Dr Noguchi). Proteins and antibodies were incubated with rotation at $4^{\circ} \mathrm{C}$ overnight, then $50 \mu$ l Protein G Mag Sepharose (Life Technologies) was added and incubated at $4{ }^{\circ} \mathrm{C}$ for $9 \mathrm{~h}$. After incubation, the supernatant was removed and beads were washed twice with TBS buffer. Proteins were eluted by adding SDS with $100 \mathrm{mM}$ DTT at $95^{\circ} \mathrm{C}$ for $5 \mathrm{~min}$.

Native-PAGE. HEK293T cells were transfected with plasmids using the same transfection ratio and protocol as in the immunocytochemistry experiment. HEK293T cells were harvested on ice and lysed in $100 \mu \mathrm{L}$ RIPA buffer without detergent. Cell lysates were separated on 4-15\% Mini-Protean TGX Gels (Bio-Rad) and transferred onto PVDF membranes. The membranes were blocked overnight and incubated with anti-FLAG or anti-EGFP antibodies diluted 1:1,000. After three washes with PBS-T, the membranes were incubated with for $1 \mathrm{~h}$ at room temperature with an anti-mouse IgG HRP-linked antibody diluted 1:5,000.

Plasma membrane protein biotinylation. HEK293T cells were transfected with plasmids using the same transfection ratio and protocol as in the immunocytochemistry experiment. HEK293T cells were washed three times with PBS and incubated twice with $0.5 \mathrm{mg} \mathrm{ml}^{-1}$ EZ-Link NHS-SS-Biotin (Pierce) for $20 \mathrm{~min}$ each. Cells were washed three times with quenching buffer $(100 \mathrm{mM}$ glycine in PBS, pH 8.0) and twice with PBS. Cells were collected and lysed in RIPA lysis buffer with complete protease inhibitor cocktail. Biotinylated proteins were precipitated overnight using $10 \mu \mathrm{l}$ Streptavidin-agarose beads with rotation at $4{ }^{\circ} \mathrm{C}$. Beads and eluates were separated by a spin column, and denatured at $95^{\circ} \mathrm{C}$ for 5 min with SDS and DTT. Samples were analysed by western blotting. Quantification of western blot bands was performed using Image J software. Full gel images of PCR and western blotting experiments can be found in Supplementary Fig. S2.
DRG isolation and electroporation. Mouse DRG cells from L4 and L5 sections were rapidly isolated and enzymatically digested. After digestion, cells were gently dissociated using fire-polished Pasteur pipettes. Cells were then seeded onto polyD-lysine pre-coated cover slips. Calcium imaging was carried out after incubation overnight. For DRG electroporation, cells were resuspended with $1 \mathrm{ml}$ PBS (-) after dissociation and $10 \mu \mathrm{g}$ mouse TRPA1b and $1 \mu \mathrm{g}$ pCMV-DsRed plasmids was electroporated into DRG by the Neon transfection system. Electroporation conditions were as follows: $900 \mathrm{~V}, 25 \mathrm{~ms}$, two pulses. Calcium imaging was carried out after incubation for $48 \mathrm{~h}$.

Calcium imaging and electrophysiology. Calcium influx was monitored with $5 \mu \mathrm{M}$ fura-2 AM. A positive response was obtained when the ratio increases by agonists were more than 0.2. The real cytosolic $\mathrm{Ca}^{2}+$ concentrations were calculated with a standard curve generated using different concentrations of extracellular $\mathrm{Ca}^{2+}$ with $5 \mu \mathrm{M}$ ionomycin ${ }^{53}$. For whole-cell patch-clamp experiments, TRPAla alone group was transfected with $0.1 \mu \mathrm{g}$ Trpala and $0.9 \mu \mathrm{g}$ empty pcDNA3.1 plasmids. TRPA1b alone group was transfected with $0.9 \mu \mathrm{g}$ Trpa $1 b$ and $0.1 \mu \mathrm{g}$ empty pcDNA3.1 plasmids. TRPA1a + TRPA1b group was transfected with $0.1 \mu \mathrm{g}$ Trpala and $0.9 \mu \mathrm{g}$ Trpalb plasmids. In the experiment that cells were transfected with different ratios, $0.1 \mu \mathrm{g}$ Trpala was transfected together with different amounts of Trpa1b and the total DNA amounts were brought to $1.1 \mu \mathrm{g}$ with empty pcDNA3.1 plasmid. The standard bath solution contained $140 \mathrm{mM} \mathrm{NaCl}, 5 \mathrm{mM} \mathrm{KCl}, 2 \mathrm{mM} \mathrm{MgCl}, 2 \mathrm{mM} \mathrm{CaCl}_{2}, 10 \mathrm{mM}$ HEPES and $10 \mathrm{mM}$ glucose, $\mathrm{pH} 7.4$, adjusted with $\mathrm{NaOH}$. The pipette solution contained $140 \mathrm{mM} \mathrm{KCl}$ $5 \mathrm{mM}$ EGTA and $10 \mathrm{mM}$ HEPES, pH 7.4, adjusted with KOH. Data were sampled at $10 \mathrm{kHz}$ and filtered at $5 \mathrm{kHz}$. The membrane potential was clamped at $-60 \mathrm{mV}$ during recording and a voltage ramp from $-100 \mathrm{mV}$ to $+100 \mathrm{mV}$ in $500 \mathrm{~ms}$ was applied for $5 \mathrm{~s}$. For inside-out single-channel patch-clamp, the pipette solution was substituted with standard bath solution, whereas the extracellular solution was changed to pipette solution used in whole-cell recording. Single-channel data were sampled at $10 \mathrm{kHz}$ and filtered at $2 \mathrm{kHz}$. Data were recorded using pClamp and analysed using Clampfit software.

Statistical analysis. Group data represent the mean \pm s.e.m. Statistical analysis was performed with Student's $t$-test or one-way ANOVA with Bonferroni's post hoc analysis. $P<0.05$ was considered to be significant.

\section{References}

1. Montell, C. \& Rubin, G. M. Molecular characterization of the Drosophila trp locus: a putative integral membrane protein required for phototransduction. Neuron 2, 1313-1323 (1989).

2. Caterina, M. J. et al. The capsaicin receptor: a heat-activated ion channel in the pain pathway. Nature 389, 816-824 (1997).

3. Story, G. M. et al. ANKTM1, a TRP-like channel expressed in nociceptive neurons, is activated by cold temperatures. Cell 112, 819-829 (2003).

4. Cordero-Morales, J. F., Gracheva, E. O. \& Julius, D. Cytoplasmic ankyrin repeats of transient receptor potential Al (TRPA1) dictate sensitivity to thermal and chemical stimuli. Proc. Natl Acad. Sci. USA 108, E1184-E1191 (2011).

5. Shigetomi, E., Tong, X., Kwan, K. Y., Corey, D. P. \& Khakh, B. S. TRPA1 channels regulate astrocyte resting calcium and inhibitory synapse efficacy through GAT-3. Nat. Neurosci. 15, 70-80 (2012).

6. Nozawa, K. et al. TRPA1 regulates gastrointestinal motility through serotonin release from enterochromaffin cells. Proc. Natl Acad. Sci. USA 106, 3408-3413 (2009).

7. Cao, D. S. et al. Expression of transient receptor potential ankyrin 1 (TRPA1) and its role in insulin release from rat pancreatic beta cells. PLoS One 7, e38005 (2012).

8. Obata, K. et al. TRPA1 induced in sensory neurons contributes to cold hyperalgesia after inflammation and nerve injury. J. Clin. Invest. 115, 2393-2401 (2005).

9. Alt, F. W. et al. Synthesis of secreted and membrane-bound immunoglobulin$\mathrm{Mu}$ heavy-chains is directed by messenger-RNAs that differ at their 3' ends. Cell 20, 293-301 (1980).

10. Early, P. et al. 2 messenger-RNAs can be produced from a single immunoglobulin- $\mu$ gene by alternative RNA processing pathways. Cell 20, 313-319 (1980).

11. Lareau, L. F., Green, R. E., Bhatnagar, R. S. \& Brenner, S. E. The evolving roles of alternative splicing. Curr. Opin. Struct. Biol. 14, 273-282 (2004).

12. Pan, Q., Shai, O., Lee, L. J., Frey, B. J. \& Blencowe, B. J. Deep surveying of alternative splicing complexity in the human transcriptome by high-throughput sequencing. Nat. Genet. 40, 1413-1415 (2008).

13. Wang, E. T. et al. Alternative isoform regulation in human tissue transcriptomes. Nature 456, 470-476 (2008).

14. Boue, S., Vingron, M., Kriventseva, E. \& Koch, I. Theoretical analysis of alternative splice forms using computational methods. Bioinformatics 18(Suppl 2): S65-S73 (2002) 
15. Rosenfeld, M. G. et al. Calcitonin mRNA polymorphism: peptide switching associated with alternative RNA splicing events. Proc. Natl Acad. Sci. USA 79, 1717-1721 (1982).

16. Kang, K. et al. Modulation of TRPA1 thermal sensitivity enables sensory discrimination in Drosophila. Nature 481, 76-80 (2012).

17. Zhong, L. et al. Thermosensory and non-thermosensory isoforms of Drosophila melanogaster TRPA1 reveal heat sensor domains of a thermoTRP channel. Cell Rep. 1, 43-55 (2012).

18. Vos, M. H. et al. TRPV1b overexpression negatively regulates TRPV1 responsiveness to capsaicin, heat and low pH in HEK293 cells. J. Neurochem. 99, 1088-1102 (2006).

19. Yildirim, E., Dietrich, A. \& Birnbaumer, L. The mouse C-type transient receptor potential 2 (TRPC2) channel: alternative splicing and calmodulin binding to its $\mathrm{N}$ terminus. Proc. Natl Acad. Sci. USA 100, 2220-2225 (2003).

20. Wang, C., Hu, H. Z., Colton, C. K., Wood, J. D. \& Zhu, M. X. An alternative splicing product of the murine trpv1 gene dominant negatively modulates the activity of TRPV1 channels. J. Biol. Chem. 279, 37423-37430 (2004).

21. Bautista, D. M. et al. TRPA1 mediates the inflammatory actions of environmental irritants and proalgesic agents. Cell 124, 1269-1282 (2006).

22. Macpherson, L. J. et al. Noxious compounds activate TRPA1 ion channels through covalent modification of cysteines. Nature 445, 541-545 (2007).

23. Boutz, P. L., Chawla, G., Stoilov, P. \& Black, D. L. MicroRNAs regulate the expression of the alternative splicing factor $\mathrm{nPTB}$ during muscle development. Genes Dev. 21, 71-84 (2007).

24. Lee, S. P. et al. Thymol and related alkyl phenols activate the hTRPA1 channel. Br. J. Pharmacol. 153, 1739-1749 (2008).

25. Fujita, F., Moriyama, T., Higashi, T., Shima, A. \& Tominaga, M. Methyl p-hydroxybenzoate causes pain sensation through activation of TRPA1 channels. Br. J. Pharmacol. 151, 153-160 (2007).

26. Karashima, Y. et al. Bimodal action of menthol on the transient receptor potential channel TRPA1. J. Neurosci. 27, 9874-9884 (2007).

27. Xiao, B. et al. Identification of transmembrane domain 5 as a critical molecular determinant of menthol sensitivity in mammalian TRPA1 channels. J. Neurosci. 28, 9640-9651 (2008).

28. Karashima, Y. et al. TRPA1 acts as a cold sensor in vitro and in vivo. Proc. Natl Acad. Sci. USA 106, 1273-1278 (2009).

29. Bandell, M. et al. Noxious cold ion channel TRPA1 is activated by pungent compounds and bradykinin. Neuron 41, 849-857 (2004).

30. McKemy, D. D., Neuhausser, W. M. \& Julius, D. Identification of a cold receptor reveals a general role for TRP channels in thermosensation. Nature 416, 52-58 (2002).

31. da Costa, D. S. et al. The involvement of the transient receptor potential A1 (TRPA1) in the maintenance of mechanical and cold hyperalgesia in persistent inflammation. Pain 148, 431-437 (2010).

32. Brierley, S. M. et al. TRPA1 contributes to specific mechanically activated currents and sensory neuron mechanical hypersensitivity. J. Physiol. 589, 3575-3593 (2011).

33. Nassini, R. et al. Oxaliplatin elicits mechanical and cold allodynia in rodents via TRPA1 receptor stimulation. Pain 152, 1621-1631 (2011).

34. Eid, S. R. et al. HC-030031, a TRPA1 selective antagonist, attenuates inflammatory- and neuropathy-induced mechanical hypersensitivity. Mol. Pain 4, 48 (2008).

35. del Camino, D. et al. TRPA1 contributes to cold hypersensitivity. J. Neurosci. 30, 15165-15174 (2010)

36. Frederick, J., Buck, M. E., Matson, D. J. \& Cortright, D. N. Increased TRPA1, TRPM8, and TRPV2 expression in dorsal root ganglia by nerve injury. Biochem. Biophys. Res. Commun. 358, 1058-1064 (2007).

37. Staaf, S., Oerther, S., Lucas, G., Mattsson, J. P. \& Ernfors, P. Differential regulation of TRP channels in a rat model of neuropathic pain. Pain 144, 187-199 (2009).

38. Persson, A. K., Xu, X. J., Wiesenfeld-Hallin, Z., Devor, M. \& Fried, K. Expression of DRG candidate pain molecules after nerve injury--a comparative study among five inbred mouse strains with contrasting pain phenotypes. J. Peripher. Nerv. Syst 15, 26-39 (2010).

39. Binder, A. et al. Transient receptor potential channel polymorphisms are associated with the somatosensory function in neuropathic pain patients. PLoS One 6, e17387 (2011)
40. Materazzi, S. et al. Cox-dependent fatty acid metabolites cause pain through activation of the irritant receptor TRPA1. Proc. Natl Acad. Sci. USA 105, 12045-12050 (2008).

41. Taylor-Clark, T. E. et al. Prostaglandin-induced activation of nociceptive neurons via direct interaction with transient receptor potential A1 (TRPA1). Mol. Pharmacol. 73, 274-281 (2008).

42. Petrus, M. et al. A role of TRPA1 in mechanical hyperalgesia is revealed by pharmacological inhibition. Mol. Pain 3, 40 (2007).

43. Tanowitz, M., Hislop, J. N. \& von Zastrow, M. Alternative splicing determines the post-endocytic sorting fate of G-protein-coupled receptors. J. Biol. Chem. 283, 35614-35621 (2008)

44. Koch, T. et al. C-terminal splice variants of the mouse mu-opioid receptor differ in morphine-induced internalization and receptor resensitization. J. Biol. Chem. 276, 31408-31414 (2001).

45. Akopian, A. N. Regulation of nociceptive transmission at the periphery via TRPA1-TRPV1 interactions. Curr. Pharm. Biotechnol. 12, 89-94 (2011).

46. Lin, S. \& Fu, X. D. SR proteins and related factors in alternative splicing. Adv. Exp. Med. Biol. 623, 107-122 (2007).

47. Hasler, R. et al. Alterations of pre-mRNA splicing in human inflammatory bowel disease. Eur. J. Cell Biol. 90, 603-611 (2011).

48. Heinhuis, B. et al. Inflammation-dependent secretion and splicing of IL32 \{gamma\} in rheumatoid arthritis. Proc. Natl Acad. Sci. USA 108, 4962-4967 (2011).

49. Eissa, N. T. et al. Alternative splicing of human inducible nitric-oxide synthase mRNA. tissue-specific regulation and induction by cytokines. J. Biol. Chem. 271, 27184-27187 (1996).

50. Ortis, F. et al. Cytokines interleukin-1beta and tumor necrosis factor-alpha regulate different transcriptional and alternative splicing networks in primary beta-cells. Diabetes 59, 358-374 (2010).

51. Hutton, J. C. \& Davidson, H. W. Cytokine-induced dicing and splicing in the beta-cell and the immune response in type 1 diabetes. Diabetes 59, 335-336 (2010).

52. Dixon, W. J. Efficient analysis of experimental observations. Annu. Rev. Pharmacol. Toxicol. 20, 441-462 (1980).

53. Everaerts, W. et al. The capsaicin receptor TRPV1 is a crucial mediator of the noxious effects of mustard oil. Curr. Biol. 21, 316-321 (2011).

\section{Acknowledgements}

We thank Dr D Julius and Dr K Noguchi for providing TRPA1 knockout mice and the polyclonal anti-TRPA1 antibody, respectively. This work was supported by grants to M.T. from the Ministry of Education, Culture, Sports, Science and Technology in Japan.

\section{Author contributions}

M.T. and Y.S. designed research and Y.Z. performed the experiments. Y.S. assisted the qRT-PCR, western blotting, immunoprecipitation, native-PAGE and biotinylation experiments. K.U. assisted the RT-PCR, molecular cloning and animal behavioural experiments. Y.Z., Y.S. and K.U. analysed the data together. Y.Z. and M.T. wrote the manuscript after discussion with the other authors.

\section{Additional information}

Accession codes: The nucleotide sequence for the Trpal splice variant reported in this paper has been deposited in GenBank under accession code KF460461.

Supplementary Information accompanies this paper at http://www.nature.com/ naturecommunications

Competing financial interests: The authors declare no competing financial interests.

Reprints and permission information is available online at http://npg.nature.com/ reprintsandpermissions/

How to cite this article: Zhou, Y. et al. Identification of a splice variant of mouse TRPA1 that regulates TRPA1 activity. Nat. Commun. 4:2399 doi: 10.1038/ncomms3399 (2013). 


\section{Publisher Correction: Identification of a splice variant of mouse TRPA1 that regulates TRPA1 activity}

Yiming Zhou, Yoshiro Suzuki, Kunitoshi Uchida \& Makoto Tominaga

Nature Communications 4:2399 doi: 10.1038/ncomms3399 (2013); Published 6 Sep 2013; Updated 29 Mar 2018

The original HTML version of this Article had an incorrect article number of 2408; it should have been 2399. This has now been corrected in the HTML; the PDF version of the Article was correct from the time of publication. 